\title{
Poly(ADP-ribose) polymerase as a novel regulator of $17 \beta$-estradiol-induced cell growth through a control of the estrogen receptor/IGF-1 receptor/PDZK1 axis
}

\author{
Hogyoung Kim, Abdelmetalab Tarhuni ${ }^{1}$, Zakaria Y Abd Elmageed ${ }^{2}$ and A Hamid Boulares ${ }^{\text {** }}$
}

\begin{abstract}
Background: We and others have extensively investigated the role of PARP-1 in cell growth and demise in response to pathophysiological cues. Most of the clinical trials on PARP inhibitors are targeting primarily estrogen receptor (ER) negative cancers with BRCA-deficiency. It is surprising that the role of the enzyme has yet to be investigated in ERmediated cell growth. It is noteworthy that ER is expressed in the majority of breast cancers. We recently showed that the scaffolding protein PDZK1 is critical for $17 \beta$-estradiol $\left(E_{2}\right)$-induced growth of breast cancer cells. We demonstrated that $\mathrm{E}_{2}$-induced PDZK1 expression is indirectly regulated by ER and requires IGF-1 receptor (IGF-1R).

Methods: The breast cancer cell lines MCF-7 and BT474 were used as ER(+) cell culture models. Thieno[2,3-c]isoquinolin-5-one (TIQ-A) and olaparib (AZD2281) were used as potent inhibitors of PARP. PARP-1 knockdown by shRNA was used to show specificity of the effects to PARP-1.

Results: In this study, we aimed to determine the effect of PARP inhibition on estrogen-induced growth of breast cancer cells and examine whether the potential effect is linked to PDZK1 and IGF-1R expression. Our results show that PARP inhibition pharmacologically by TIQ-A or olaparib or by PARP-1 knockdown blocked $E_{2}$-dependent growth of MCF-7 cells. Such inhibitory effect was also observed in olaparib-treated BT474 cells. The effect of PARP inhibition on cell growth coincided with an efficient reduction in $\mathrm{E}_{2}$-induced PDZK1 expression. This effect was accompanied by a similar decrease in the cell cycle protein cyclin D1. PARP appeared to regulate $\mathrm{E}_{2}$-induced PDZK1 at the mRNA level. Such regulation may be linked to a modulation of IGF-1R as PARP inhibition pharmacologically or by PARP-1 knockdown efficiently reduced $\mathrm{E}_{2}$-induced expression of the receptor at the protein and $\mathrm{mRNA}$ levels.
\end{abstract}

Conclusions: Overall, our results show for the first time that PARP regulates $E_{2}$-mediated cell growth by controlling the ER/IGF-1R/PDZK1 axis. These findings suggest that the relationship between ER, PDZK1, and IGF-1R may be perturbed by blocking PARP function and that PARP inhibitors may be considered in clinical trials on ER(+) cancers.

Keywords: PARP, 17/-estradiol, Gene regulation, Cell growth, Breast cancer, PDZK1, IGF-1R, MCF-7 cells

\section{Background}

It is well established that estrogen receptor (ER) is expressed in the majority of breast cancers and are responsive to standard therapy with tamoxifen as the leading drug [1]. Despite the success of this therapy,

\footnotetext{
*Correspondence: hboulr@lsuhsc.edu

${ }^{1}$ The Stanley Scott Cancer Center, Louisiana State University Health

Sciences Center, 1700 Tulane Ave, New Orleans, LA 70112, USA

Full list of author information is available at the end of the article
}

reduction of breast cancer recurrence is only by $50 \%$ and an even lower reduction in mortality rate $(\sim 30 \%)[1,2]$. Accordingly, finding new therapeutic targets is urgently needed. Achieving this goal requires the identification of new players in the complex process of the disease. We have recently shown that PDZK1, a scaffold protein, plays an important role in estrogen-induced growth of breast cancer cells and demonstrated a strong correlation between the expression of the protein and human 
breast malignancy [3]. We also reported that PDZK1 gene expression is not a direct product of ER stimulation; rather, it requires the expression and function of IGF-1 receptor (IGF-1R) [3]. PDZK1 appears to harbor oncogenic activity and promote cell growth by enhancing EGFR-stimulated MEK/ERK1/2 signaling and IGFinduced Akt phosphorylation [4]. Interestingly, PDZK1 plays this important role through stabilization of the integrity of Akt, Her2/Neu, and EGFR [4]. The co-chaperone Cdc37 appears to play an important role in PDZK1mediated stability of Akt [4]. These aforementioned findings demonstrated a novel relationship between PDZK1, Akt, Her2/Neu, EGFR and Cdc37 in breast cancer unraveling a new axis that can be targeted therapeutically to reduce the burden of human breast cancer.

Poly (ADP-ribose) polymerase (PARP)-1, a member of the PARP family of proteins, has initially been described as a DNA repair enzyme playing primarily as a regulatory protein controlling traffic of DNA repair proteins during base excision repair [5,6]. A prominent function of this enzyme is in cell death both as an effector and as a substrate to some of the caspases [7]. We demonstrated many years ago that cleavage of PARP- 1 is critical for the normal progression of the apoptotic process and that interference with such cleavage enhances cell death and may even cause a switch to necrosis $[7,8]$. Increasing evidence from our laboratory and many others demonstrate an important role for this enzyme in tissue injury associated with oxidative stress and inflammation including asthma and atherosclerosis [8-13]. PARP-1 is thought to participate in inflammation by regulating the expression of several inflammatory factors including adhesion molecules, TNF- $\alpha$, interleukins, and inducible nitric oxide synthase (iNOS) most of which are controlled by NF-kB (4). PARP inhibitors have shown great potential against breast and ovarian cancers especially those with BRCA mutations [14]. The combination of PARP inhibitors with DNA damaging chemotherapeutic drugs have shown to induce the specific demise of BRCA-deficient cancer cells leading to a synthetic lethality phenotype while sparing the life of normal cells [15]. Many clinical trials have demonstrated efficacy of PARP inhibitors and their potential as therapeutic strategy that can be utilized in the clinic $[14,15]$. However, the focus on BRCA-deficient breast cancer prevented the examination of the effects of PARP inhibitors on ER positive breast cancer cells and, as a result, may be reducing the full therapeutic potential of these drugs.

In the present study we wished to determine the effect of PARP inhibition pharmacologically or PARP-1 knockdown on estrogen-induced growth of the ER positive breast cancer cell line MCF-7 and BT474 and to examine whether the potential effect was related to a modulation of $\mathrm{E}_{2}$-induced PDZK1 expression.

\section{Methods \\ Materials}

DMEM, penicillin, streptomycin, and fetal bovine serum (FBS) were purchased from Invitrogen (Camarillo, CA, USA). Charcoal/dextran-treated FBS (CDSS) was purchased from Hyclone (Logan, Utah, USA); 17 $\beta$-Estradiol $\left(\mathrm{E}_{2}\right)$, and the PARP-1 inhibitor TIQ-A were from SigmaAldrich (St. Louis, MO, USA); olaparib (AZD2281) was from Selleckchem (Pittsburgh, PA, USA); IGF-1R inhibitor AG1024 was from Calbiochem (San Diego, CA, USA); PARP-1 and PDZK-1 shRNA expressing lentiviral vectors and the control virus were from Santa Cruz Biotechnology (Santa Cruz, CA, USA). Unless otherwise indicated, all other drugs were purchased from Sigma-Aldrich.

\section{Cell culture, cell proliferation, cell survival, transfection, immunoblot analysis, and RT-PCR}

The ER positive breast Cancer cell line MCF-7 was obtained from ATCC (Manassas, VA, USA). The second ER positive breast cancer cell line BT474 was originally obtained from ATCC but provided by Dr. Wanguo Liu (LSUHSC). The two cell lines were cultured according to ATCC instructions. These cell lines are authenticated by ATCC using short tandem repeat (STR) profiling. This PCR-based approach permits the authentication of human cell lines with high resolution down to the individual donor. Upon receipt from ATCC, the morphology was confirmed by microscopy and population-doubling times were determined using the trypan blue dye exclusion method. After cells reached $70 \%$ confluence, complete DMEM medium was changed to DMEM supplemented with 5\% CDSS followed by $E_{2}(1 \mathrm{nM})$ treatment for the indicated time periods. Cells were also treated with TIQ-A, olaparib, or AG1024. Cell proliferation was measured by MTT assay after 2 days of treatment as previously described [3]. In some experiments, cells were transduced with a lentiviral vector encoding control shRNA or shRNA targeting human PARP-1 or PDZK1 (Santa Cruz Biotechnology) according to the manufacturer's instructions. Cells were selected with puromycin dihydrochloride (Santa Cruz Biotechnology) and treated as described above. After the treatments, cells were collected and subjected to total RNA or protein preparation. Isolated RNA was reverse-transcribed and the resulting cDNA was subjected to conventional or quantitative PCR with primer sets purchased from IDT (San Jose, CA, USA) specific to human PDZK1, IGF-1R and $\beta$-actin as described [3]. Protein extracts were subjected to immunoblot analysis with antibodies to PDZK1 (EPR3751 clone) (Novus Biological), PARP-1 (BD Biosciences, San Jose, CA), cyclin D (Santa Cruz Biotechnology) or GADPH (Santa Cruz Biotechnology). The immunoblots were incubated with the appropriate secondary antibodies and signals were detected by ECL (Pierce, Rockford, IL, USA). 


\section{Statistical analysis}

Data are presented as mean \pm SEM from at least three separate experiments. Comparisons between multiple groups were performed with one-way ANOVA with Bonferroni's test using GraphPad software, Version 5 (La Jolla, CA, USA). Statistical significance was considered at $p<0.05$.

\section{Results and discussion}

PARP inhibition blocks $E_{2}$-dependent growth of MCF-7 cells Figure 1a shows that PARP inhibition with the specific inhibitor TIQ-A exerted no effect on the growth of the breast cancer cell line MCF-7 except at a very high concentration of $50 \mu \mathrm{M}$. This result is consistent with our previous report which concluded that the growth inhibition caused by the high concentrations of PARP inhibitors is most likely associated with toxicity of the drugs rather than an effect related to PARP inhibition [16, 17]. However, it is now established that PARP inhibition causes cell death when combined with BRCA deficiency [18]. It is rather surprising that the role of PARP in estrogen-mediated growth of breast cancer cells has not been investigated. We thus wished to examine whether PARP-1 plays a role in growth mediated by $E_{2}$. Figure $1 b$ shows that $E_{2}$ treatment, as expected, induced $\sim 40 \%$ increase in growth of MCF-7 cells, which is typical as reported by our laboratory and others $[3,19]$. PARP inhibition by TIQ-A blocked $E_{2}$-induced growth of MCF-7 cells even at a concentration as low as $0.5 \mu \mathrm{M}$. The specificity of this effect was confirmed using a PARP-1 shRNA-mediated knockdown approach (Figure $1 c, d)$. These results clearly show that PARP plays a role in $\mathrm{E}_{2}$-mediated cell growth and that its inhibition can be regarded as a means to block abnormal proliferation of ER positive breast cancer cells. Such inhibition was achieved with rather low concentrations of the PARP inhibitor. Our results show that in addition to the ability of PARP inhibitor to promote a synthetic lethality phenotype in BRCAdeficient cells [18], it can also reduce $\mathrm{E}_{2}$-dependent growth in ER positive tumor cells.
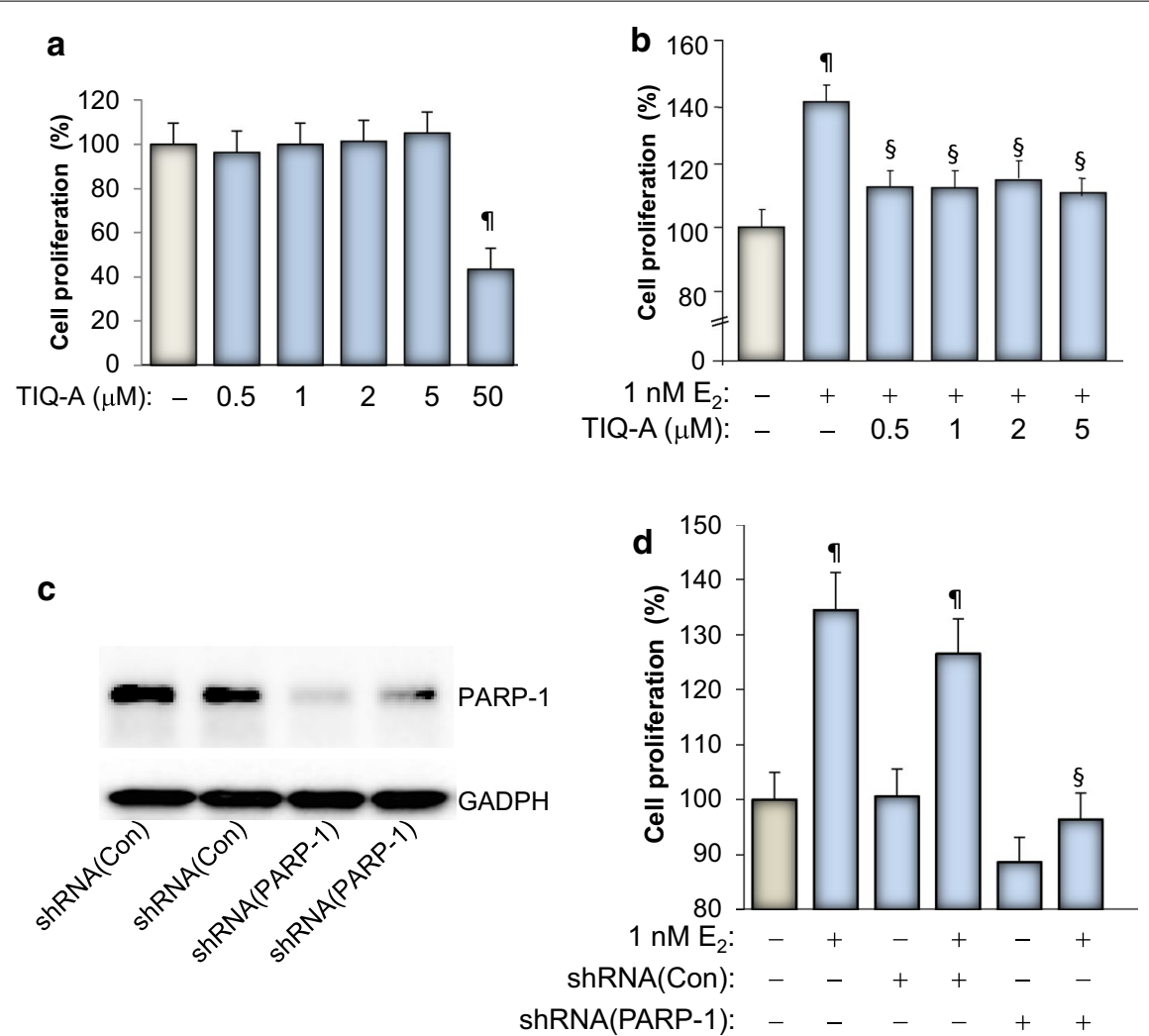

Figure 1 Effect of PARP inhibition on $\mathrm{E}_{2}$-stimulated growth of MCF-7 cells. a MCF-7 cells were treated for $48 \mathrm{~h}$ in the absence or presence of increasing concentrations of TIQ-A. Cell viability was assessed using a MTT assay. "Difference from viability values of cells that did not receive TIQ-A; $p<0.05$. b MCF-7 cells were stimulated with $1 \mathrm{nM} \mathrm{E}_{2}$ for $48 \mathrm{~h}$ in the absence or presence of the indicated TIQ-A concentrations. Cell viability was then assessed using a MTT assay. "Difference from viability values of cells that did not receive TIQ-A; $p<0.05 .{ }^{.}$Difference from viability values of cells that were treated with $\mathrm{E}_{2}$ alone; $p<0.05$. c MCF-7 cells were transduced with a viral vector encoding control shRNA or a shRNA targeting human PARP-1. Protein extracts were prepared and subjected to immunoblot analysis with antibodies against PARP-1 or GAPDH. $\mathbf{d}$ Cells expressing control or PARP-1-targeting shRNA were treated with $\mathrm{E}_{2}$ for $48 \mathrm{~h}$ after which viability was assessed as described above. "Difference from respective untreated controls; ${ }^{\S}$ difference from $\mathrm{E}_{2}$-treated cells; $p<0.05$. 
PARP inhibition-associated reduction in $\mathrm{E}_{2}$-induced growth of MCF-7 cells is linked to a reduction in PDZK1 expression with a concomitant decrease in cyclin D1

We recently showed in two detailed studies that $\mathrm{E}_{2}$-mediated cell growth can be governed by PDZK1 [4].
We thus speculated that PARP-1 may be involved in ERmediated growth of MCF-7 cells potentially through a control of PDZK1 expression. As reported previously [3] and as shown in Figure 2a, knockdown of PDZK1 blocks growth of MCF-7 cells in response to stimulation with

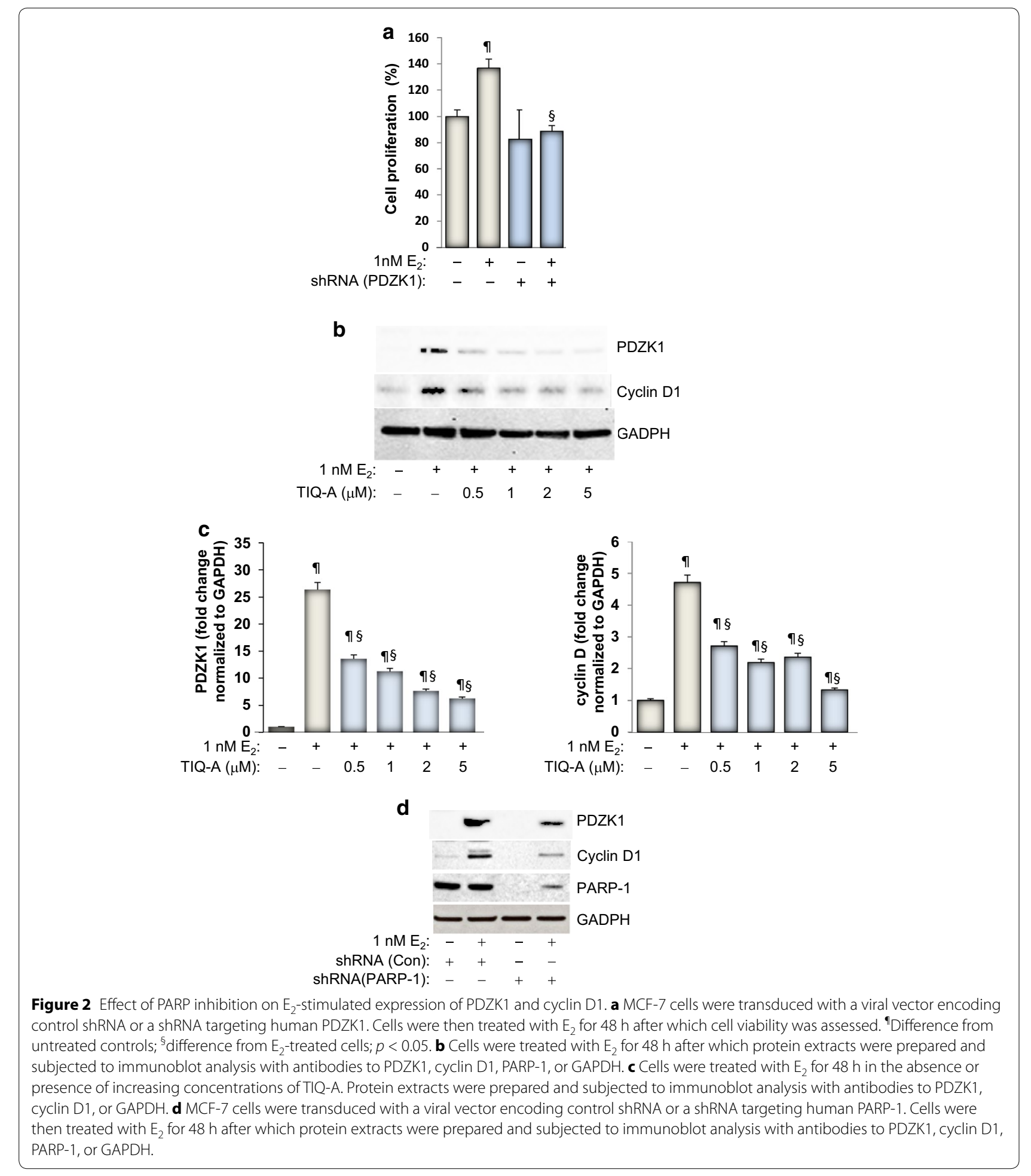


$1 \mathrm{nM}$ of $\mathrm{E}_{2}$. We next wished to examine whether PARP-1 enzymatic activity or protein expression influences $E_{2}-$ induced PDZK1 expression. To this end, MCF-7 cells were exposed to $\mathrm{E}_{2}$ for $48 \mathrm{~h}$ in the absence or presence of increasing concentrations of TIQ-A. Immunoblot analysis of collected cell extracts showed that PARP inhibition was very efficient in reducing expression of PDZK1 (Figure 2b, c). The results also show that partial reduction of PDZK1 by $0.5 \mu \mathrm{M}$ TIQ-A was sufficient to completely block $E_{2}$-induced growth of MCF-7 cells (as shown in Figure 1b). These results are consistent with our previous observation that partial knockdown of $E_{2}$ induced PDZK1 was sufficient to completely block $E_{2}$ induced growth of MCF-7 cells [3]. The involvement of PDZK1 in growth of MCF-7 cells appears to be linked to expression of cyclin D1 as knockdown of PDZK1 prevents $E_{2}$-induced expression of the cell cycle protein [3, 4]. Although it is unlikely that PARP is always required for the expression of cyclin D1, it was recently reported that the expression of the enzyme as well as its activity are required for cyclin D1 expression in response to ectopically-expressed Krüppel-like factor 8 (KLF8) [20]. An additional link between PARP-1 and cyclin D1 is the relationship of both proteins to NF- $\kappa$ B. We and others have shown that PARP-1 plays an important role in $\mathrm{NF}-\kappa \mathrm{B}$ function $[21,22]$. Furthermore, cyclin D1 expression can be controlled by NF-kB [23]. We next examined whether the role of PARP-1 in PDZK1 expression influences expression of cyclin D1 upon $E_{2}$ exposure. Figure $2 \mathrm{~d}$ shows that PARP inhibition with TIQ-A reduced cyclin D1 expression in a manner similar to that exerted on PDZK1. Additionally, knockdown of PARP-1 markedly reduced expression of PDZK1 and cyclin D1 showing the specificity of the effect (Figure 2d). It is noteworthy that an increase in PDZK1 expression in response to $E_{2}$ treatment did not change the levels of PARP-1 (Figure 2d). This is consistent with our published results showing that ectopic PDZK1 expression in the absence of $E_{2}$ exposure does not affect expression of PARP-1 [4]. Although these results do not prove that

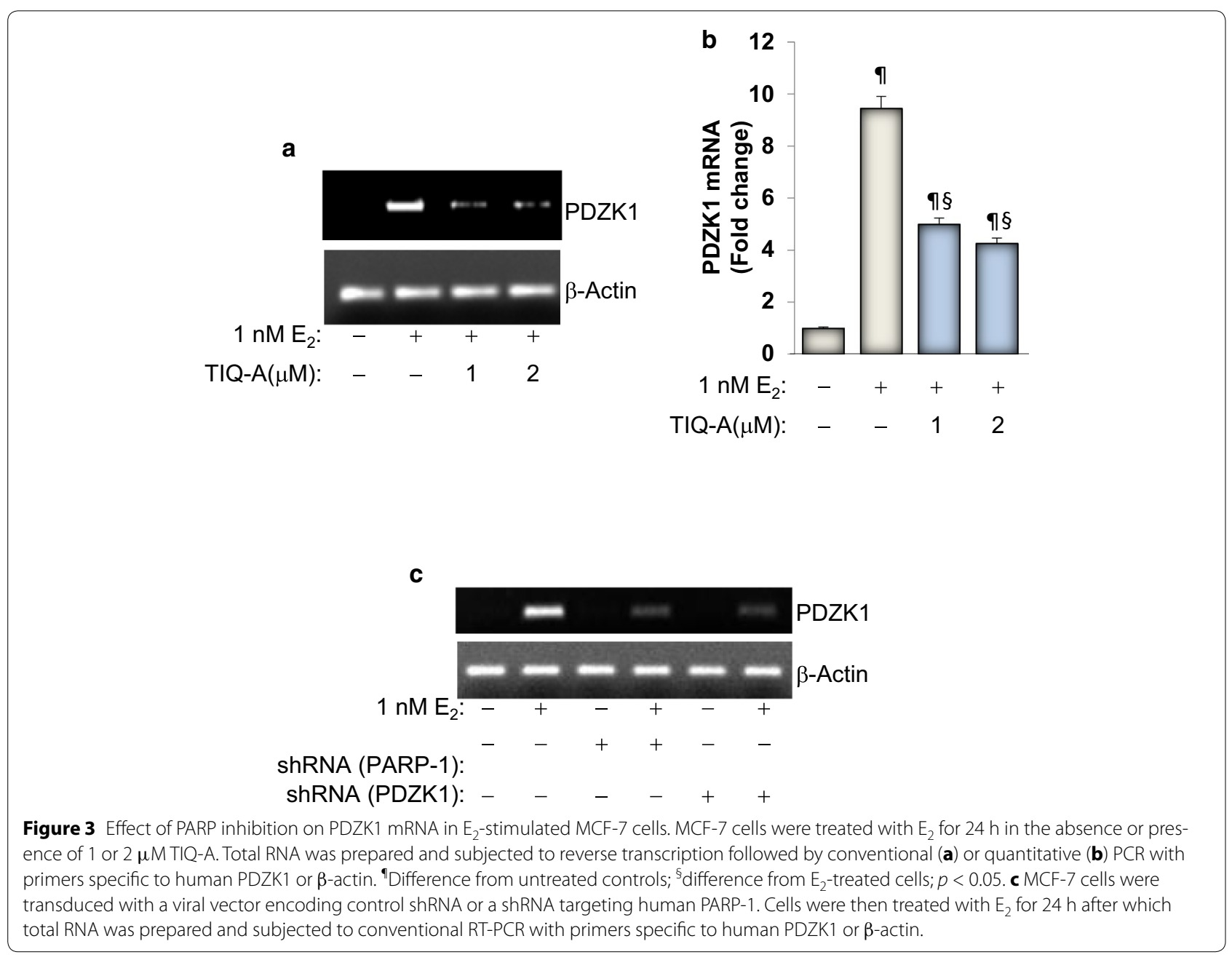


$\mathrm{E}_{2}$ exposure does not affect the activity of PARP, we can, however, conclude that it does not change the expression levels of this DNA repair enzyme.

\section{PARP regulates $E_{2}$-induced PDZK1 at the mRNA level} We have shown previously that PARP-1 may control expression of a number of proteins by regulating their expression at the mRNA level or by influencing their stability. For instance, PARP-1 regulates expression of several inflammatory proteins including adhesion molecules, iNOS, TNF- $\alpha$, IL-1 $\beta$, IL-5, and IL-13 [12, 24, 25]. PARP-1 may also regulate proteins at the level of their integrity. Indeed, PARP-1 influences the fate of STAT- 6 upon IL-4 or allergen exposure by preventing the degradation of the transcription factor by calpains [26]. We therefore wished to determine whether PARP-1 regulates expression of PDZK1 at the level of mRNA. Figure 3a shows that PARP inhibition was very effective in blocking $E_{2}$-mediated increase in PDZK1 transcript. The effect of the PARP inhibitor on expression of $\mathrm{E}_{2}$-induced PDZK1 mRNA was confirmed by quantitative RT-PCR (Figure 3b). Consistent with these results, PARP-1 knockdown was equally efficient in blocking $\mathrm{E}_{2}$-mediated increase in PDZK1 mRNA (Figure 3c). These results suggest that PARP-1 may regulate PDZK1 expression at the level of mRNA. However, it is premature to conclude that it influences its gene transcription. Given the lack of evidence for the control of PDZK1 by proteolysis, it is unlikely that PARP-1 regulates the protein by influencing its integrity.

PARP-1 regulates $\mathrm{E}_{2}$-mediated growth of MCF-7 cells and PDZK1 expression by controlling the expression of IGF-1R

We reported that PDZK1 expression was indirectly regulated by ER- $\alpha$ activation, requiring IGF-1R expression and function [3]. Blocking the activity of IGF-1R by the specific inhibitor AG1024 blocked $\mathrm{E}_{2}$-induced PDZK1 in MCF-7 cells (Figure 4a). We thus speculated that PARP-1 may be regulating PDZK1 expression through IGF-1R. Indeed, PARP-1 inhibition with TIQ-A reduced expression of $E_{2}$-induced IGF-1R even at the lowest concentration (Figure $4 \mathrm{~b}$ ). Figure $4 \mathrm{c}$ shows that PARP inhibition efficiently blocked $E_{2}$-induced expression of IGF-1R mRNA in MCF-7 cells. PARP-1 knockdown almost completely blocked the expression of $\mathrm{E}_{2}$-induced IGF-1R (Figure $4 d$ ) confirming the results attained using the PARP inhibitor and demonstrating specificity of the effect.

PARP inhibition by olaparib (AZD2281) blocks $E_{2}$-mediated growth of MCF-7 and BT474 cells and inhibits expression of PDZK1 and IGF-1R

An important step in proving the validity of targeting PARP to control growth of $\mathrm{ER}(+)$ breast cancer cells is

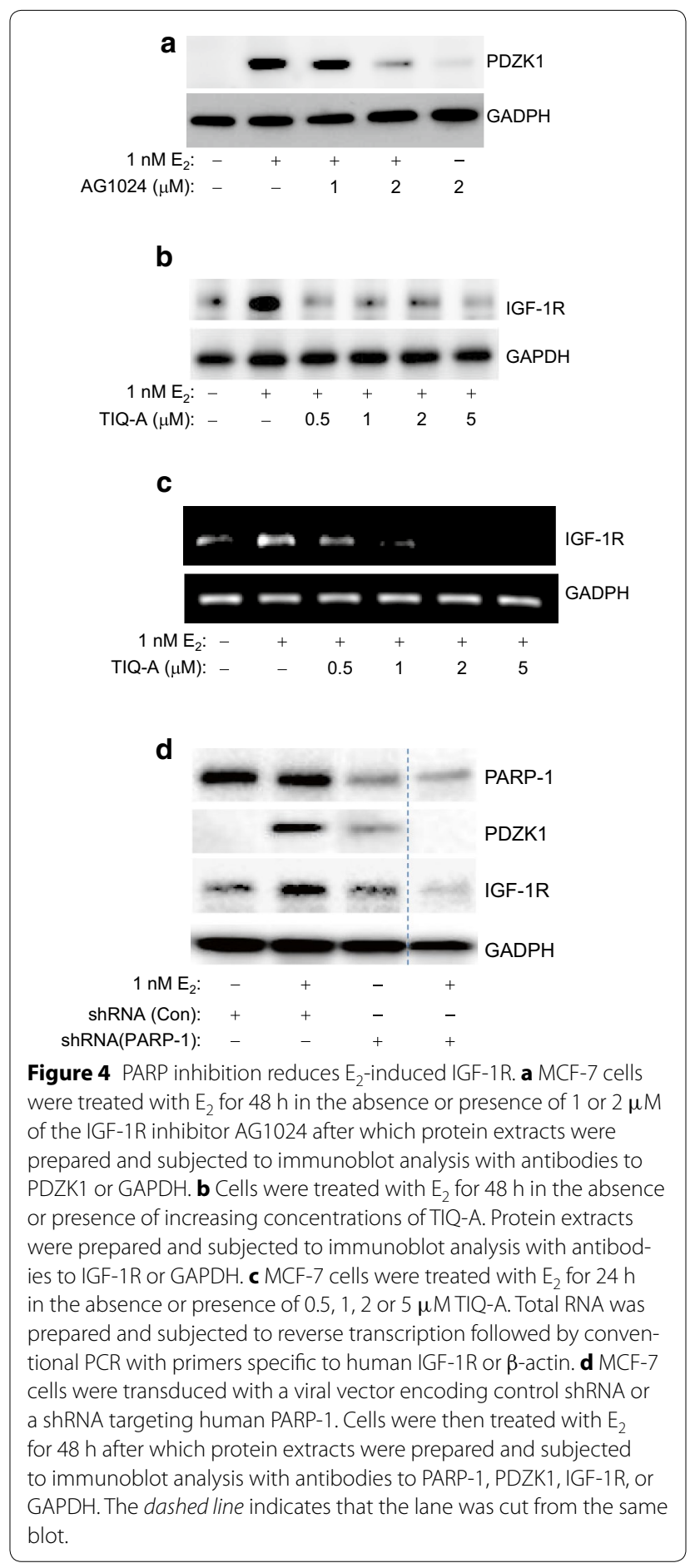

to demonstrate that PARP inhibitors other than TIQ-A can achieve the same effect and to show that such effect is observed in breast cancer cells other than MCF-7 cells. Olaparib (AZD2281), a potent PARP inhibitor, is currently used in a large number of clinical trials targeting $\mathrm{BRCA}(-)$ breast and ovarian cancers [15]. We thus 
tested the effect of different concentrations of olaparib on growth of MCF-7 cells. Figure 5a shows that olaparib at the $5 \mu \mathrm{M}$ concentration partially but significantly reduced $\mathrm{E}_{2}$-induced cell growth; the $10 \mu \mathrm{M}$ concentration of the drug completely blocked growth of the treated cells. Figure $5 \mathrm{~b}$ shows that olaparib was very effective in blocking growth of BT474 cells, another ER(+) cell line [27].

We next examined whether growth inhibition of MCF-7 cells by olaparib correlated with a decrease in PDZK1, cyclin D1 and IGF-1R. Figure 5c clearly shows that olaparib treatment markedly blocked expression of $E_{2}$-induced PDZK1 in a manner similar to that achieved by TIQ-A. Such inhibition of PDZK1 expression occurred concomitantly with a reduction in cyclin D1 and IGF-1R upon exposure to $E_{2}$ in MCF-7 cells. Figure $5 \mathrm{~d}$ shows that PARP inhibition reduces PDZK1 and IGF-1R at the level of mRNA. Overall, these results are consistent with those attained using TIQ-A as a PARP inhibitor and demonstrate that the ability of PARP inhibition to block $\mathrm{E}_{2}$-induced growth is not limited to TIQ-A or MCF-7 cells but may be extended to other ER(+) breast cancer cells.

\section{Conclusions}

It is well recognized that breast and ovarian cancer heterogeneity continues to represent a major obstacle in tailoring precise and efficient therapies with minimal toxicities. Much effort is needed to identify key determining factors that control the growth of cancer cells. Accordingly, insights on the role of new factors or identification of new functions for already established players may provide us with new directions and strategies with which cancer can be retarded or blocked. Collectively, our results suggest that PARP may play an important role in $E_{2}$-induced cell growth by regulating the expression of PDZK1 through a control of the stimulated expression of IGF-1R. To our knowledge, our results are the first in a

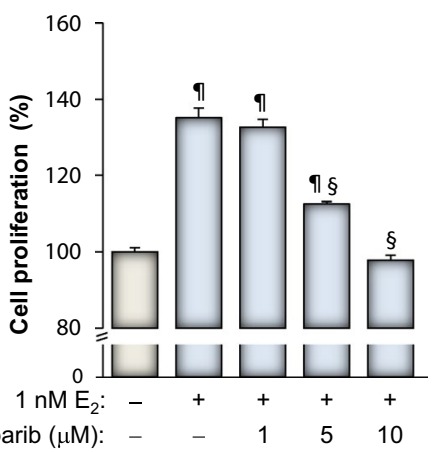

C

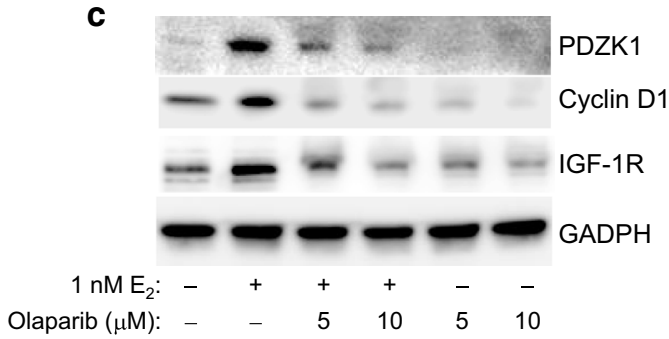

b

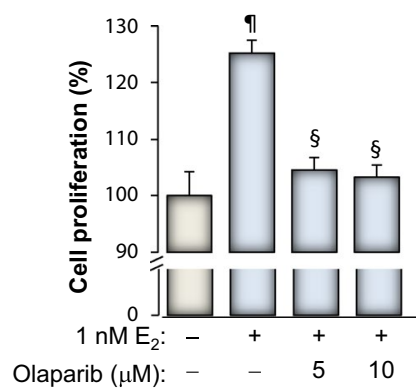

d

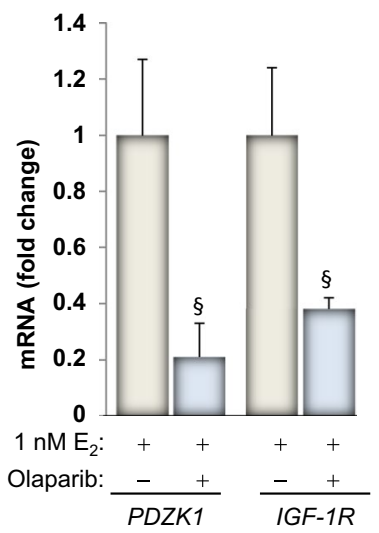

Figure 5 Olaparib treatment blocks $E_{2}$-mediated growth of MCF-7 and BT474 cells and inhibits expression of PDZK1, IGF-1R, and cyclin D1. MCF-7 (a) or BT474 (b) cells were stimulated with $1 \mathrm{nM} \mathrm{E}_{2}$ for $48 \mathrm{~h}$ in the absence or presence of the indicated olaparib concentrations. Cell viability was then assessed using a MTT assay. "Difference from viability values of cells that did not receive olaparib; $p<0.05$. ${ }^{5}$ Difference from viability values of cells that were treated with $E_{2}$ alone; $p<0.05$. c MCF-7 cells were treated with $E_{2}$ for $48 \mathrm{~h}$ in the absence or presence of increasing concentrations of olaparib after which protein extracts were prepared and subjected to immunoblot analysis with antibodies to PDZK1, cyclin D1, IGF-1R, or GAPDH. d MCF-7 cells were treated with $E_{2}$ for $24 \mathrm{~h}$ in the presence of $5 \mu \mathrm{M}$ olaparib. Total RNA was prepared and subjected to reverse transcription followed by quantitative PCR with primers specific to human PDZK1, IGF-1R or $\beta$-actin. ${ }^{\S}$ Difference from $\mathrm{E}_{2}$-treated cells; $p<0.05$. 
showing a connection between PARP and ER-stimulated growth and that such trait may be considered in the current effort in establishing PARP inhibitors in the treatment of breast and ovarian cancers. We have shown a strong relationship between PDZK1 and IGF-1R expression in human breast cancer and that PDZK1 may be a determinant in breast tumorigenesis [3, 4]. The molecular link between PDZK1 and IGF-1R was supported by a significant correlation between protein and mRNA levels of the two factors in two independent cohorts of human breast cancer tissues [3].

The current results suggest that the relationship between ER, PDZK1, and IGF-1R may be an appealing axis to be targeted by PARP inhibitors. It is noteworthy that a great deal of effort is being spent focusing primarily on triple negative breast and ovarian cancers with a BRCA deficiency to specifically achieve synthetic lethality of cancer cells with the BRCA mutation [28]. Although the presented results do not provide a complete understanding of the mechanism by which PARP regulates PDZK1 or IGF-1R, they support the hypothesis that PARP inhibitors, including olaparib, may be useful for treatment of $\mathrm{ER}(+)$ and estrogen-dependent cancers. Obviously, more work is needed to validate such hypothesis and unravel the molecular mechanisms underlying the cross-talk between the different factors.

\section{Abbreviations}

PARP: poly(ADP-ribose) polymerase; ER: estrogen receptor; IGF-1R: IGF-1 receptor; TIQ-A: thieno[2,3-c]isoquinolin-5-one.

\section{Authors' contributions \\ $\mathrm{KH}$ conducted most of the experiments, participated in data analysis, and assisted in the writing of the manuscript. AT conducted some of the experi- ments and participated in data analysis. AZ participated in the planning of the experiments, participated in data analysis and the writing of the manuscript. AHB conceived the study, participated in data analysis, provided the funding for the study and wrote the manuscript. All authors read and approved the final manuscript.}

\section{Author details}

${ }^{1}$ The Stanley Scott Cancer Center, Louisiana State University Health Sciences Center, 1700 Tulane Ave, New Orleans, LA 70112, USA. ${ }^{2}$ Tulane University Cancer Center, New Orleans, LA 70112, USA.

\section{Acknowledgements}

We would like to thank the members of the Boulares'lab for proofreading the manuscript. This work was supported, in part, by Grant RSG-116608 from the American Cancer Society and Grant HL072889 from the NIH as well as funds from the Louisiana Cancer Research Consortium (New Orleans, LA) to H. Boulares.

\section{Compliance with ethical guidelines}

\section{Competing interests}

The authors declare that they have no competing interests.

Received: 10 January 2015 Accepted: 29 June 2015

Published online: 17 July 2015

\section{References}

1. Early Breast Cancer Trialists' Collaborative G, Godwin J, Gray R, Clarke M, Cutter D, Darby S, McGale P, Pan HC, Taylor C et al (2011) Relevance of breast cancer hormone receptors and other factors to the efficacy of adjuvant tamoxifen: patient-level meta-analysis of randomised trials. Lancet 378:771-784

2. Mehta A, Tripathy D (2014) Co-targeting estrogen receptor and HER2 pathways in breast cancer. Breast 23:2-9

3. Kim H, Abd Elmageed ZY, Ju J, Naura AS, Abdel-Mageed AB, Varughese S, Paul D, Alahari S, Catling A, Kim JG, Boulares AH (2013) PDZK1 is a novel factor in breast cancer that is indirectly regulated by estrogen through IGF-1R and promotes estrogen-mediated growth. Mol Med 19:253-262

4. Kim H, Abd Elmageed ZY, Davis C, El-Bahrawy AH, Naura AS, Ekaidi I, Abdel-Mageed AB, Boulares AH (2014) Correlation between PDZK1, Cdc37, Akt and breast cancer malignancy: the role of PDZK1 in cell growth through Akt stabilization by increasing and interacting with Cdc37. Mol Med 20:270-279

5. Shall S (1992) Poly ADP-ribosylation reaction. Jpn J Cancer Res 83

6. Shall S, de Murcia G (2000) Poly(ADP-ribose) polymerase-1: what have we learned from the deficient mouse model? Mutat Res 460:1-15

7. Boulares AH, Yakovlev AG, Ivanova V, Stoica BA, Wang G, lyer S, Smulson M (1999) Role of poly(ADP-ribose) polymerase (PARP) cleavage in apoptosis. Caspase 3-resistant PARP mutant increases rates of apoptosis in transfected cells. J Biol Chem 274:22932-22940

8. Hans CP, Zerfaoui M, Naura AS, Catling A, Boulares AH (2008) Differential effects of PARP inhibition on vascular cell survival and ACAT-1 expression favouring atherosclerotic plaque stability. Cardiovasc Res 78:429-439

9. Virag L, Szabo C (2002) The therapeutic potential of poly(ADP-Ribose) polymerase inhibitors. Pharmacol Rev 54:375-429

10. Pacher P, Szabo C (2008) Role of the peroxynitrite-poly(ADP-ribose) polymerase pathway in human disease. Am J Pathol 173:2-13

11. Hans CP, Zerfaoui M, Naura AS, Troxclair D, Strong JP, Matrougui K, Boulares AH (2009) Thieno[2,3-c]isoquinolin-5-one, a potent poly(ADP-ribose) polymerase inhibitor, promotes atherosclerotic plaque regression in high-fat diet-fed apolipoprotein E-deficient mice: effects on inflammatory markers and lipid content. J Pharmacol Exp Ther 329:150-158

12. Oumouna-Benachour $K$, Hans CP, Suzuki Y, Naura A, Datta R, Belmadani S, Fallon K, Woods C, Boulares AH (2007) Poly(ADP-ribose) polymerase inhibition reduces atherosclerotic plaque size and promotes factors of plaque stability in apolipoprotein E-deficient mice: effects on macrophage recruitment, nuclear factor-kappaB nuclear translocation, and foam cell death. Circulation 115:2442-2450

13. Mukhopadhyay P, Horvath B, Kechrid M, Tanchian G, Rajesh M, Naura AS, Boulares AH, Pacher P (2011) Poly(ADP-ribose) polymerase-1 is a key mediator of cisplatin-induced kidney inflammation and injury. Free Radic Biol Med 51:1774-1788

14. Curtin NJ, Szabo C (2013) Therapeutic applications of PARP inhibitors: anticancer therapy and beyond. Mol Aspects Med 34:1217-1256

15. Jelinic P, Levine DA (2014) New insights into PARP inhibitors' effect on cell cycle and homology-directed DNA damage repair. Mol Cancer Ther 13:1645-1654

16. Inbar-Rozensal D, Castiel A, Visochek L, Castel D, Dantzer F, Izraeli S, Cohen-Armon M (2009) A selective eradication of human nonhereditary breast cancer cells by phenanthridine-derived polyADP-ribose polymerase inhibitors. Breast Cancer Res 11:R78

17. Kim H, Naura AS, Errami Y, Ju J, Boulares AH (2011) Cordycepin blocks lung injury-associated inflammation and promotes BRCA1-deficient breast cancer cell killing by effectively inhibiting PARP. Mol Med

18. Bryant HE, Schultz N, Thomas HD, Parker KM, Flower D, Lopez E, Kyle S, Meuth M, Curtin NJ, Helleday T (2005) Specific killing of BRCA2-deficient tumours with inhibitors of poly(ADP-ribose) polymerase. Nature 434:913-917

19. Ptak A, Gut P, Blachuta M, Rak A, Gregoraszczuk EL (2009) Direct inhibition of ERK1/2 phosphorylation as a possible mechanism for the antiproliferative action of 3,4-diOH-PCB3 in the MCF-7 cell line. Toxicol Lett 190:187-192

20. Lu H, Wang X, Li T, Urvalek AM, Yu L, Li J, Zhu J, Lin Q, Peng X, Zhao J (2011) Identification of poly (ADP-ribose) polymerase-1 (PARP-1) as a novel Kruppel-like factor 8-interacting and -regulating protein. J Biol Chem 286:20335-20344 
21. Zerfaoui M, Suzuki Y, Naura AS, Hans CP, Nichols C, Boulares AH (2008) Nuclear translocation of p65 NF-kappaB is sufficient for VCAM-1, but not ICAM-1, expression in TNF-stimulated smooth muscle cells: differential requirement for PARP-1 expression and interaction. Cell Signal 20:186-194

22. Zerfaoui M, Errami Y, Naura AS, Suzuki Y, Kim H, Ju J, Liu T, Hans CP, Kim JG, Abd Elmageed ZY et al (2010) Poly(ADP-ribose) polymerase-1 is a determining factor in $\mathrm{Crm} 1$-mediated nuclear export and retention of p65 NF-kappa B upon TLR4 stimulation. J Immunol 185:1894-1902

23. Hinz M, Krappmann D, Eichten A, Heder A, Scheidereit C, Strauss M (1999) NF-kappaB function in growth control: regulation of cyclin D1 expression and G0/G1-to-S-phase transition. Mol Cell Biol 19:2690-2698

24. Oumouna M, Datta R, Oumouna-Benachour K, Suzuki Y, Hans C, Matthews K, Fallon K, Boulares H (2006) Poly(ADP-ribose) polymerase-1 inhibition prevents eosinophil recruitment by modulating Th2 cytokines in a murine model of allergic airway inflammation: a potential specific effect on IL-5. J Immunol 177:6489-6496

25. Naura AS, Datta R, Hans CP, Zerfaoui M, Rezk BM, Errami Y, Oumouna M, Matrougui K, Boulares AH (2009) Reciprocal regulation of iNOS and PARP-1 during allergen-induced eosinophilia. Eur Respir J 33:252-262

26. Datta R, Naura AS, Zerfaoui M, Errami Y, Oumouna M, Kim H, Ju J, Ronchi VP, Haas AL, Boulares AH (2011) PARP-1 deficiency blocks IL-5 expression through calpain-dependent degradation of STAT- 6 in a murine asthma model. Allergy 66:853-861

27. Holliday DL, Speirs V (2011) Choosing the right cell line for breast cancer research. Breast Cancer Res 13:215

28. Sonnenblick A, de Azambuja E, Azim HA Jr, Piccart M (2015) An update on PARP inhibitors-moving to the adjuvant setting. Nat Rev Clin Oncol 12:27-41
Submit your next manuscript to BioMed Central and take full advantage of:

- Convenient online submission

- Thorough peer review

- No space constraints or color figure charges

- Immediate publication on acceptance

- Inclusion in PubMed, CAS, Scopus and Google Scholar

- Research which is freely available for redistribution

Submit your manuscript at

www.biomedcentral.com/submit 